\title{
Corrosion des aciers inoxydables par les solutions d'eau de javel
}

\author{
par \\ L. KERHERVE, G. DAUFIN et Françoise MICHEL
}

\section{Ré s u m é}

La présence d'ions chlorure $\left(\mathrm{Cl}^{-}\right)$dans les solutions d'eau de javel est responsable de l'existence de potentiels électrochimiques critiques de corrosion caverneuse et de corrosion par piqûres sur les aciers inoxydables. Les ions hypochlorite $\left(\mathrm{Cl0}^{-}\right)$interviennent comme agents oxydants qui élèvent le potentiel électrochimique libre jusqu'à des valeurs égales ou supérieures aux potentiels critiques, provoquant ainsi la corrosion spontanée de l'acier. La suppression, depuis la fin de 1976, de l'addition d'ions dichromate $\left(\mathrm{Cr}_{2} \mathrm{O}_{7}{ }^{2}\right)$ n'entraîne aucune modification du pouvoir corrosif des solutions d'eau de javel.

A l'état poli mécanique $(80 \mu \mathrm{m})$, les aciers ferritiques du type $17 \% \mathrm{Cr}$ et les aciers austénitiques du type $18 \% \mathrm{Cr} 10 \% \mathrm{Ni}$ présentent une résistance à la corrosion tout à fait comparable. L'addition de titane ou de molybdène et surtout de ces 2 éléments simultanément, renforce la résistance à la corrosion aussi bien des aciers ferritiques que des aciers austénitiques.

Nous avons défini, pour les aciers inoxydables à $18 \% \mathrm{Cr} 10 \% \mathrm{Ni}$, un large domaine de couples température-durée de contact, où les solutions d'eau de javel n'entraînent aucune corrosion. Ce domaine est d'autant plus restreint que la concentration est plus élevée $\left(100,300,1000 \mathrm{mg} \mathrm{Cl} \mathrm{Cl}_{2} \mathrm{l}^{-1}\right)$. L'absence d'agitation réduit également le domaine sans corrosion. Enfin, les durées de contact possibles sans apparition de la corrosion sont d'autant plus courtes que la température est plus élevée.

Mots clés :

Corrosion caverneuse - Corrosion par piqûres - Aciers inoxydables - Eau de javel - Hypochlorite - Industrie alimentaire.

Institut National de la Recherche Agronomique, Laboratoire de Recherches de Technologie Laitière, 65, rue de Saint-Brieuc - 35042 Rennes cedex. 


\title{
Su $\mathrm{m} m$ a ry
}

\author{
CORROSION OF STAINLESS STEELS \\ BY COMMERCIAL SODIUM HYPOCHLORITE SOLUTIONS
}

Chloride ions $\left(\mathrm{Cl}^{-}\right)$are responsible for a critical electrochemical potential of crevice and pitting corrosion when stainless steels are in contact with sodium hypochlorite solutions. Hypochlorite ions $\left(\mathrm{ClO} \mathrm{O}^{-}\right)$only act as oxidizing agents: they increase the free electrochemical potential of stainless steel up to values equal or higher than that of the critical potential, inducing spontaneous corrosion of steels. Since 1976, potassium dichromate is no more added to commercial hypochlorite extracts but the corrosive power of solution is not modified.

Mechanically polished ferritic (17\% chromium type) and austenitic (18\% chromium - $10 \%$ nickel type) stainless steels show similar corrosion resistance. Addition of molybdenum or titanium or especially both improve resistance of both types of steels.

For $18 \%$ chromium - $10 \%$ Nickel stainless steel (AISI 304 L) we have defined a wide area of couples temperature-contact time where hypochlorite solutions do not induce any corrosion. The higher the concentration $\left(100,300,1000 \mathrm{mg} \mathrm{Cl}_{2} \cdot \mathrm{l}^{-1}\right)$, the smaller the area. Absence of agitation also reduces aera without corrosion. Finally, the higher the temperature (up to $90^{\circ} \mathrm{C}$ ), the shorter the time to corrosion.

Key words:

Crevice corrosion - Pitting corrosion - Stainless steels - Sodium hypochlorite Food industry.

\section{INTRODUCTION}

L'eau de javel est largement utilisée pour la désinfection du matériel alimentaire, en raison de son excellente efficacité bactéricide (Sainclivier, 1964) et de son coût modeste. Malheureusement, ce produit peut être à l'origine de corrosions localisées du matériel, dont les plus fréquentes sont la corrosion caverneuse et la corrosion par piqûres. La première se développe dans des interstices (espace entre métal et joint, entre métal et métal aux points de contact de plaques d'échangeurs, sous dépôt ou tartre,...); la seconde sur les surfaces nues. Peu de travaux ont été consacrés à la corrosion des aciers inoxydables par les solutions commerciales d'hypochlorite de sodium : Botham (1962), Daufin et al., (1977 a, 1979 et 1981) pour la corrosion caverneuse; Speight (1947) ; Botham et Dummet 1949) ; Botham (1962) ; Rossman (1964) ; Shere et al., (1965) ; Cowan (1971) ; Daufin (1977); Daufin et al., (1977 a, 1979 et 1981) pour la 
corrosion par piqûres. Ces travaux n'indiquent malheureusement pas à l'utilisateur dans quelles conditions ces solutions n'entraînent pas de corrosion. Des précautions d'emploi draconiennes ont par contre été données (Féd. Int. Lait., 1980). Par ailleurs, depuis septembre 1976, les eaux de javel françaises ne doivent plus contenir de dichromate de potassium, oxydant puissant, qui pouvait avoir une part importante de responsabilité dans le pouvoir corrosif des solutions.

Nous avons voulu, dans ce travail, actualiser les connaissances concernant la corrosion de divers aciers inoxydables en contact avec des solutions d'eau de javel et surtout préciser les conditions limites de leur utilisation dans le contexte de l'industrie alimentaire.

\section{MATERIEL ET METHODES}

\section{Aciers inoxydables utilisés}

Les nuances $1,5,6,7$ et 8 de structure ferritique ne contiennent pas de nickel; les nuances $15,19,20,23$ et 28 sont des aciers austénitiques (tab. 1). Les aciers 15 et 19 , du type $18 \mathrm{Cr} 10 \mathrm{Ni}$, de provenance et d'élaboration différentes, se différencient par la teneur en soufre, la nature et le nombre des inclusions non métalliques (Daufin et al., 1979). Les échantillons $(100 \times 50 \times 2 \mathrm{~mm})$ étaient découpés dans des tôles industrielles commerciales laminées à froid, ayant subi le traitement thermique classique : recuit de normalisation à 800 $900^{\circ} \mathrm{C}$ pour les aciers ferritiques, hypertrempe à $1050-1100^{\circ} \mathrm{C}$ pour les aciers austénitiques. Afin d'éviter l'apparition de corrosion préférentielle sur les tranches, celles-ci étaient polies au papier abrasif de granulométrie $12 \mu \mathrm{m}$ puis surpassivées (Defranoux, 1968). Les faces des échantillons étaient polies sous courant d'eau au papier abrasif de granulométrie $80 \mu \mathrm{m}$ puis passivées à l'air pendant $30 \mathrm{~min}$. L'aire d'épreuve (\# $50 \mathrm{~cm}^{2}$ ) était délimitée à l'aide de vernis " lacomit "* afin d'éviter toute corrosion parasite au niveau de la ligne d'eau. La corrosion par piqûres était étudiée sur des échantillons nus, la corrosion caverneuse sur des échantillons munis d'un dispositif de caverne artificielle représenté sur la figure 1. L'aire de la caverne était d'environ $0,5 \mathrm{~cm}^{2}$, celle de la surface nue d'environ $50 \mathrm{~cm}^{2}$.

\section{Solutions corrosives}

Les extraits commerciaux d'eau de javel contiennent de l'hypochlorite de sodium $\left(\mathrm{Na}^{+}, \mathrm{Cl}^{-}\right)$, du chlorure de sodium $\left(\mathrm{Na}^{+}, \mathrm{Cl}^{-}\right)$, de la soude et des traces de chlorate de sodium $\left(\mathrm{Na}^{+}, \mathrm{ClO}_{3}{ }^{-}\right)$. Au

\footnotetext{
* Lacomit N 2567 A - Durferrit électrosels.
} 
TABLEAU 1 - TABLE 1

Composition chimique des alliages utilisés (\% massique)

Chemical composition of alloys (mass \%)

\begin{tabular}{|c|c|c|c|c|c|c|c|c|c|c|}
\hline \multirow{2}{*}{$\begin{array}{c}\text { Alliage } \\
\text { Alloy }\end{array}$} & \multirow{2}{*}{$\begin{array}{c}\text { Repère } \\
\text { mark }\end{array}$} & \multicolumn{2}{|c|}{ Référence } & \multirow{2}{*}{$\mathrm{C}$} & \multirow{2}{*}{$\mathrm{Cr}$} & \multirow{2}{*}{$\mathrm{Ni}$} & \multirow{2}{*}{ Mo } & \multirow{2}{*}{$\mathrm{Ti}$} & \multirow{2}{*}{$\mathrm{Mn}$} & \multirow{2}{*}{$\mathrm{S}$} \\
\hline & & AFNOR & AISI & & & & & & & \\
\hline $\begin{array}{l}17 \mathrm{Cr} \\
17 \mathrm{Cr} \mathrm{Ti} \\
17 \mathrm{Cr} \mathrm{Mo} \\
18 \mathrm{Cr} 1 \mathrm{MoTi} \\
19 \mathrm{Cr} 2 \mathrm{MoTi} \text { (a) }\end{array}$ & $\begin{array}{l}1 \\
6 \\
5 \\
7 \\
8\end{array}$ & $\begin{array}{c}\text { Z4C17 } \\
\text { Z4CT17 } \\
\text { Z6CD17 } \\
\text { Z5CDT18 } \\
\text { Z2CDT19 }\end{array}$ & $\begin{array}{l}430 \\
430 \mathrm{Ti} \\
434 \\
434 \mathrm{Ti} \\
434 \mathrm{Ti}\end{array}$ & $\begin{array}{l}0,041 \\
0,038 \\
0,055 \\
0,047 \\
0,019\end{array}$ & $\begin{array}{l}16,24 \\
16,86 \\
16,49 \\
17,50 \\
19,70\end{array}$ & $\begin{array}{l}0,16 \\
0,20 \\
0,18 \\
0,29 \\
0,27\end{array}$ & $\begin{array}{l}0,05 \\
0,07 \\
0,97 \\
1,09 \\
2,5\end{array}$ & $\begin{array}{l}0,005 \\
0,63 \\
0,005 \\
0,71 \\
0,525\end{array}$ & $\begin{array}{l}0,36 \\
0,34 \\
0,42 \\
0,43 \\
0,16\end{array}$ & $\begin{array}{l}0,006 \\
0,012 \\
0,007 \\
0,008 \\
0,010\end{array}$ \\
\hline $\begin{array}{llll}18 & \mathrm{Cr} & 10 \mathrm{Ni} & \text { (b) } \\
18 \mathrm{Cr} & 10 \mathrm{Ni} & \text { (b) } \\
18 \mathrm{Cr} & 10 \mathrm{NiTi} \\
17 \mathrm{Cr} & 12 \mathrm{Ni} 2 \mathrm{Mo} \\
17 \mathrm{Cr} & 12 \mathrm{Ni} 2 \mathrm{MoTi}\end{array}$ & $\begin{array}{l}15 \\
19 \\
20 \\
23 \\
28\end{array}$ & $\begin{array}{c}\text { Z2CN18-10 } \\
\text { Z2CN18-10 } \\
\text { Z6CNT18-10 } \\
\text { Z2CND17-12 } \\
\text { Z8CNDT17-12 }\end{array}$ & $\begin{array}{l}304 \mathrm{~L} \\
304 \mathrm{~L} \\
321 \\
316 \mathrm{~L} \\
316 \mathrm{Ti}\end{array}$ & $\begin{array}{l}0,025 \\
0,021 \\
0,044 \\
0,026 \\
0,067\end{array}$ & $\begin{array}{l}18,00 \\
18,30 \\
17,96 \\
16,85 \\
17,42\end{array}$ & $\begin{array}{r}9,90 \\
10,13 \\
9,13 \\
12,15 \\
11,56\end{array}$ & $\begin{array}{l}0,013 \\
0,11 \\
0,14 \\
2,18 \\
2,16\end{array}$ & $\begin{array}{l}0,003 \\
0,010 \\
0,39 \\
-\overline{0,39}\end{array}$ & $\begin{array}{l}1,50 \\
1,65 \\
1,67 \\
1,61 \\
1,62\end{array}$ & $\begin{array}{l}0,010 \\
0,006 \\
0,005 \\
0,005 \\
0,008\end{array}$ \\
\hline
\end{tabular}

(a) Acier industriel expérimental à basse teneur en éléments interstitiels (C, N).

(b) Aciers de même nuance mais de provenance et d'élaboration différentes. 


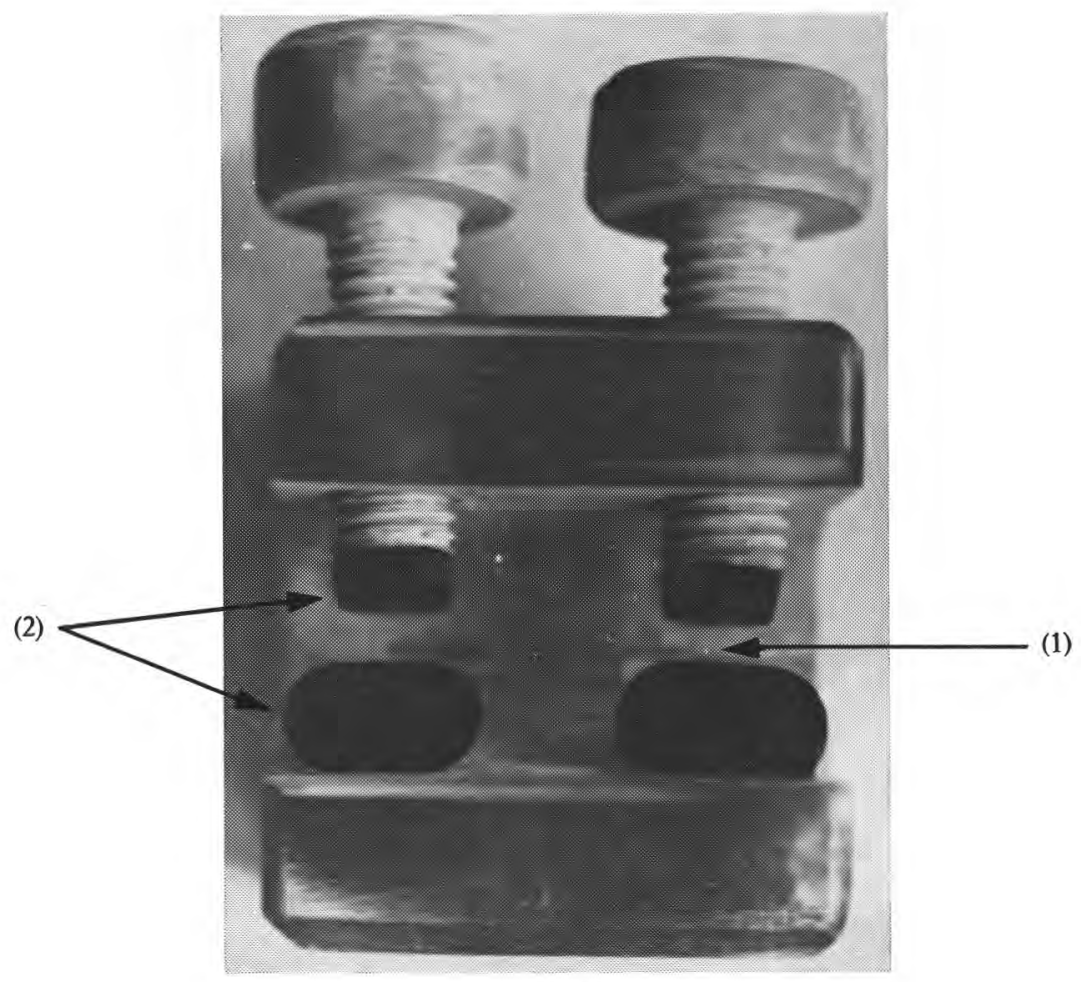

Fig. 1

Dispositif de caverne artificielle

Device for crevice corrosion

(1) Emplacement de l'échantillon en acier inoxydable.

Place for sample of stainless steel.

(2) Joints en caoutchouc nitrile d'échangeur.

Pieces of nitrile rubber gaskets from plate of heat exchanger.

cours du temps et en fonction des conditions de conservation (lumière, température), l'hypochlorite se décompose en oxygène natif, chlorure $\left(\mathrm{Cl}^{-}\right)$et éventuellement chlorate $\left(\mathrm{ClO}_{3}^{-}\right)$et chlorite $\left(\mathrm{ClO}_{2}^{-}\right)$ (Castang et al., 1965).

L'influence des ions présents dans les extraits de javel était étudiée en utilisant différentes solutions dont les caractéristiques figurent dans le tableau 2. Les solutions d'eau de javel A et B, de préparation récente, ne se différenciaient que par l'addition de 0,75 mg. $1^{-1}$ de dichromate $\left(\mathrm{Cr}_{2} \mathrm{O}_{7}{ }^{2-}\right)$ dans $\mathrm{B}$, concentration corres- 
TABLEAU 2 - TABLE 2

Composition des solutions corrosives d'essai

Composition of corrosive test solutions

\begin{tabular}{|c|c|c|c|c|c|}
\hline \multirow{2}{*}{$\begin{array}{l}\text { Solutions corrosives } \\
\text { (corrosive solutions) }\end{array}$} & \multicolumn{5}{|c|}{ Teneur (mg.1-1) en/(Concentration (mg.1-1) in) } \\
\hline & $\mathrm{C} 10^{-*}$ & $\mathrm{Cl}^{-}$ & $\begin{array}{r}\mathrm{ClO}_{3}^{-} \\
+\mathrm{ClO}_{2}^{-}\end{array}$ & $\mathrm{OH}^{-}$ & $\mathrm{Cr}_{2} \mathrm{O}_{7}^{2-}$ \\
\hline \multicolumn{6}{|l|}{$\begin{array}{l}\text { Eau de javel } \\
\text { (Sodium hypochlorite) }\end{array}$} \\
\hline $\begin{array}{l}\text { A J de préparation récente } \\
\text { B Y recently prepared } \\
\text { C vieillie - aged }\end{array}$ & $\begin{array}{r}435,2 \\
435,2 \\
2,03\end{array}$ & $\begin{array}{l}174,8 \\
174,8 \\
278,5\end{array}$ & $\begin{array}{r}15,3 \\
15,3 \\
131,1\end{array}$ & $\begin{array}{l}8,3 \\
8,3 \\
8,3\end{array}$ & $\begin{array}{l}0 \\
0,73 \\
0\end{array}$ \\
\hline \multicolumn{6}{|l|}{ Modèles/(model) } \\
\hline $\begin{array}{l}\mathrm{D} \\
\mathrm{E} \\
\mathrm{F}\end{array}$ & $\begin{array}{l}0 \\
0 \\
0\end{array}$ & $\begin{array}{l}174,8 \\
278,5 \\
174,8\end{array}$ & $\begin{array}{c}15,3 \\
131,1 \\
0\end{array}$ & $\begin{array}{l}8,3 \\
8,3 \\
8,3\end{array}$ & $\begin{array}{l}0 \\
0 \\
0\end{array}$ \\
\hline
\end{tabular}


pondant approximativement à celle existant dans l'eau de javel avant septembre 1976. La solution d'eau de javel E était préparée par vieillissement de l'extrait commercial, à température ambiante, jusqu'à décomposition quasi-complète de l'hypochlorite. Les solutions modèles $\mathrm{D}, \mathrm{E}$ et $\mathrm{F}$, préparées à partir de produits chimiques, ne contenaient pas d'hypochlorite. Si l'on excepte cet élément, D et $\mathrm{E}$ étaient identiques à $\mathrm{A}$ et $\mathrm{B}$ respectivement, $\mathrm{F}$ étant une solution témoin de chlorure de sodium.

L'étude des conditions limites d'utilisation des solutions désinfectantes était réalisée en employant plusieurs lots d'extraits commerciaux d'eau de javel titrant entre 36,7 et 46,7 degrés chlorométriques**. L'emploi de tels extraits donne des résultats statistiquement identiques, à condition d'ajuster la concentration des solutions d'essai à la teneur voulue en hypochlorite (exprimée tout au long du texte en $\mathrm{mg} \mathrm{Cl}_{2} \cdot \mathrm{1}^{-1}$ ). Ces solutions étaient préparées par dilution dans l'eau de ville (teneur en $\mathrm{NaCl}$ voisine de $58 \mathrm{mg} . \mathrm{l}^{-1}$ ). En cas d'agitation, elle était assurée par la rotation d'un barreau aimanté tournant à environ 125 t.min ${ }^{-1}$.

\section{Méthode d'étude de la corrosion}

Les critères de résistance à la corrosion par piqûres et à la corrosion caverneuse et les méthodes permettant de les déterminer ont fait l'objet d'une analyse critique approfondie (Daufin et al., 1982). Le tracé des variations du potentiel électrochimique de l'alliage en fonction du temps $(\mathrm{E}=\mathrm{f}(\mathrm{t}))$ permet de déterminer les durées avant apparition de la corrosion (fig. 2.1) : caverneuse (t cav) et par piqûres $(\mathrm{t} p)$. Cette dernière forme de corrosion est généralement précédée de la formation de piqûres repassivées après une durée $\mathrm{t}$ pr, qui peuvent endommager la surface du matériau (Daufin et al., 1982). Dans certains essais, en l'absence de corrosion spontanée (fig. 2.2.1), la courbe $E=f(t)$ était interrompue après $2 \mathrm{~h}$ et on mesurait le potentiel libre à ce moment $(2 \mathrm{~h})$. On traçait alors les courbes intensité-potentiel $(\mathrm{i}=\mathrm{f}(\mathrm{E})$ à une vitesse de balayage du potentiel de $6 \mathrm{mV} \cdot \mathrm{min}^{-1}$ (fig. 2.2.2), jusqu'à obtention des potentiels critiques de corrosion caverneuse (Ecav), de piqûres repassivées (Epr) et de piqûres (Ep). Plus la différence entre et chacun de ces trois potentiels critiques est grande, moins le risque de corrosion est grand. Tous les potentiels étaient repérés par rapport à l'électrode au calomel saturé (E.C.S.). Chaque essai était répété 5 fois et nous donnons, soit l'étendue des variations entre les valeurs extrêmes si nous effectuons des comparaisons, soit la valeur minimale relative à ces 5 mesures si nous évaluons une résistance absolue à la corrosion.

** Un degré chlorométrique correspond à $3,17 \mathrm{~g}$ de chlore $\left(\mathrm{Cl}_{2}\right)$ par litre. 
2.1. Apparition spontanée de la corrosion: courbes potentiel électrochimique en fonction du temps $(E=f(t))$
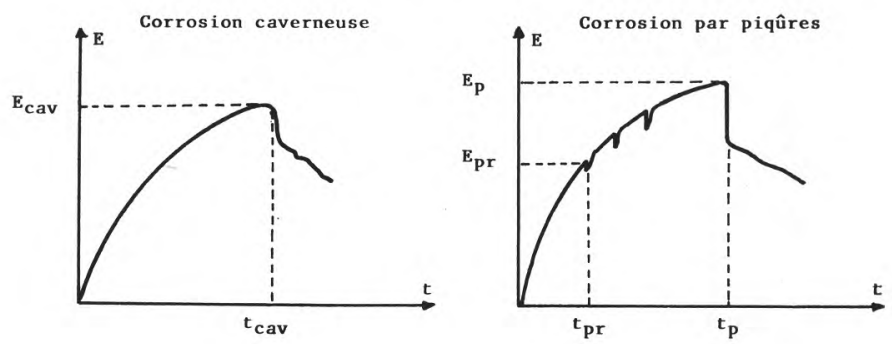

2.2. Absence de corrosion spontanée au cours de l'essai

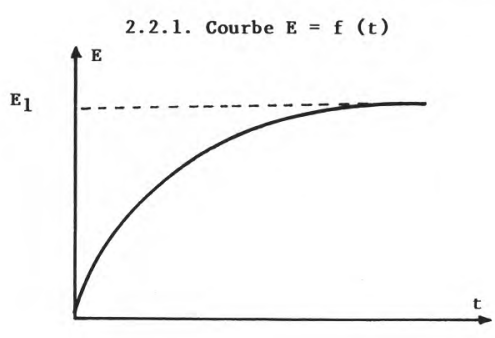

2.2.2. Courbe intensité-potentiel $(i=f(E))$
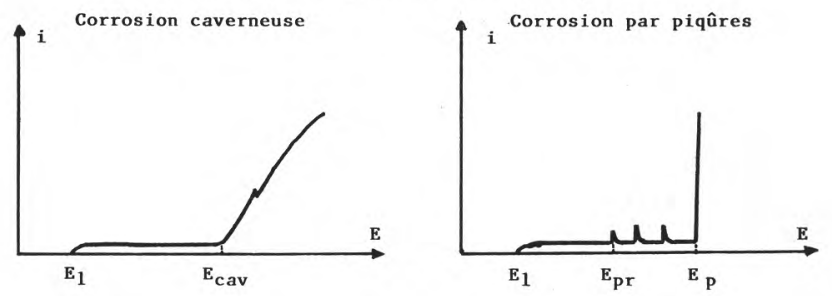

Fig. 2

Courbes utilisées pour déterminer les valeurs des critères de résistance à la corrosion

Potentiel de corrosion caverneuse, Ecav; de piqûres repassivées, Epr ; de piqûres, $\mathrm{Ep}$; libre $\mathrm{E}_{1}$; durée avant corrosion caverneuse, tcav ; par piqûres repassivées, $\mathrm{t}$ pr ; par piqûres, $\mathrm{t} \mathrm{p}$.

Curves used for determining value of corrosion resistance criteria Potential for crevice corrosion, Ecav; repassivated pits Epr; pitting corrosion Ep; free potential, $E_{l}$; time to crevice corrosion, $t$ cav; repassivated pits, tpc; pitting corrosion, $t p$. 

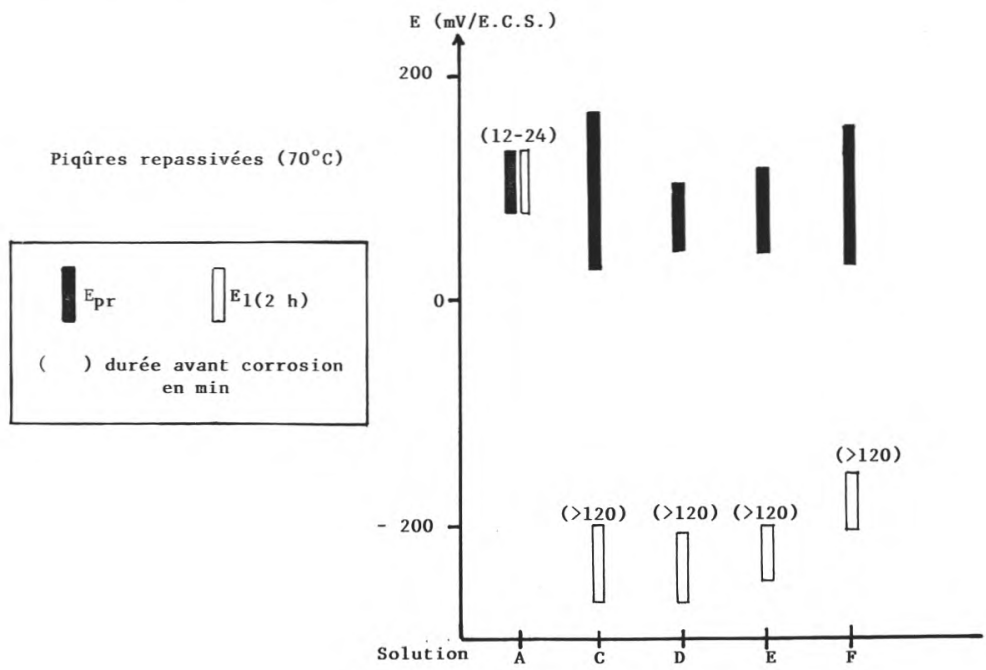

Corrosion caverneuse $\left(50^{\circ} \mathrm{C}\right)$

\section{E (mV/E.C.S.)}

200

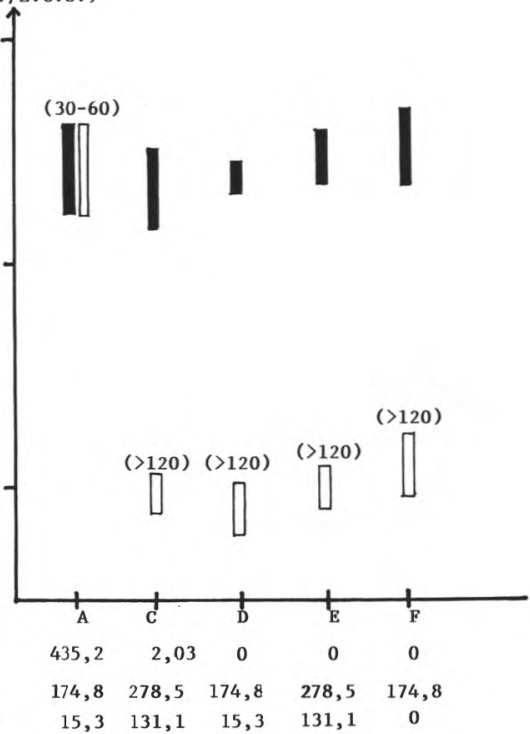

Fig. 3

Influence de la nature des ions présents dans les solutions corrosives sur la corrosion de l'acier inoxydable $18 \mathrm{Cr} 10 \mathrm{Ni}$ (repère 15) (poli au papier đe granulométrie $80 \mu \mathrm{m}$, passivé à l'air pendant $30 \mathrm{~min}$ ).

Potentiel de corrosion caverneuse, Ecav ; de piqûres repassivées, Epr ; libre après $2 \mathrm{~h}$ d'immersion, $\mathrm{E}_{1}(2 \mathrm{~h})$.

Influence of nature of ions in corrosive solutions on corrosion of $304 \mathrm{~L}$ stainless steel (mark 15) (polished with paper of $80 \mu \mathrm{m}$ granulometry; passivated in air during $30 \mathrm{~min}$ ).

Potential for crevice corrosion, Ecav; repassivated pits, Epr; free potential after $2 \mathrm{~h}$ immersion, $E_{l}(2 \mathrm{~h})$. 
Tous les essais étaient réalisés par simple immersion dans la solution corrosive. Ce type d'essais, très facile à mettre en œuvre, est en effet aussi sévère, parfois plus, que des essais en immersionémersion alternées reproduisant les conditions de contacts discontinus rencontrées industriellement entre le matériau et les liquides corrosifs (succession de contacts de quelques minutes à quelques heures avec le produit alimentaire, les solutions de rinçage, de nettoyage et de désinfection et avec l'atmosphère ambiante) (Daufin et al., 1982).

A l'issue des essais, les échantillons faisaient systématiquement l'objet d'une observation au microscope de métallographie.

\section{RESULTATS}

Les valeurs des potentiels critiques (Ecav et Epr) de l'acier inoxydable Z2CN 18-10 (repère 15) mesurées dans les cinq solutions, sont indépendantes de la nature et de la teneur des ions présents dans les différentes solutions corrosives A, C, D, E, et F (fig. 3). Il en est de même des valeurs du potentiel libre, à l'exception de celle mesurée dans la solution $\mathrm{A}$, de préparation récente, nettement plus élevée que dans les autres, et qui atteint d'ailleurs, après quelques minutes, la valeur de Ecav et de Epr, entraînant ainsi

Fig. 4

Influence de l'addition de dichromate de potassium à la solution d'eau de javel sur la durée avant corrosion de l'acier inoxydable $18 \mathrm{Cr} 10 \mathrm{Ni}$ (repère 15) (poli au papier de granulométrie $80 \mu \mathrm{m}$, passivé 30 min à l'air.

$\mathrm{t}$ cav, $\mathrm{t} \mathrm{pr}, \mathrm{t} \mathrm{p}$ : durée avant corrosion caverneuse, par piqûres repassivées, par piqûres.

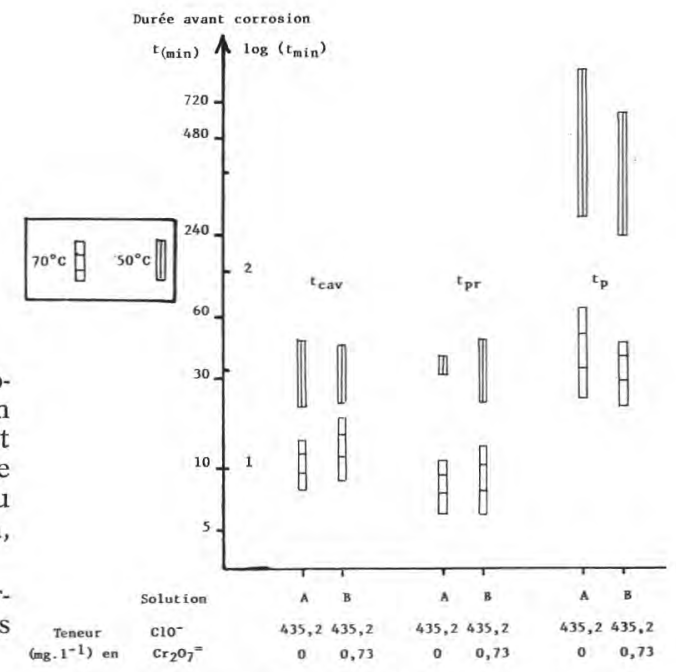

Influence of addition of potassium dichromate to sodium hypochlorite solution on time to corrosion of $304 \mathrm{~L}$ stainless steel (mark 15) (polished with paper of $80 \mu \mathrm{m}$ granulometry, passivated in air during $30 \mathrm{~min}$ ).

t cav, t pr, $t$ p: time to crevice corrosion, repassivated pits, pitting corrosion. 

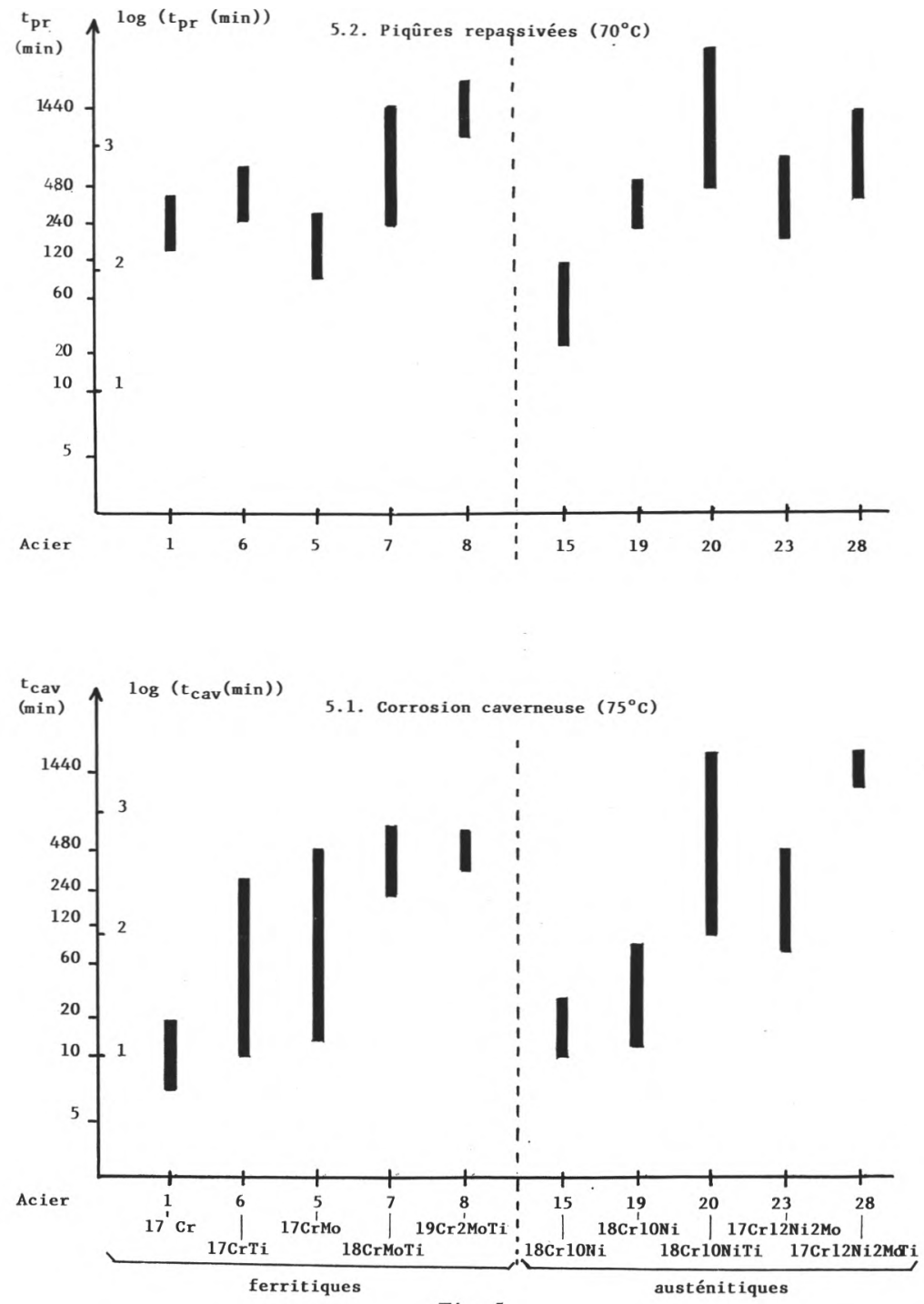

Fig. 5

Durée avant corrosion de divers aciers inoxydables dans la solution agitée d'eau de javel à $300 \mathrm{mg} \mathrm{Cl} \mathrm{Cl}^{-1}$ (polis au papier de granulométrie $80 \mu \mathrm{m}$; passivés à l'air pendant $30 \mathrm{~min}$ ).

Time to corrosion for some stainless steels in agitated sodium hypochlorite solution at $300 \mathrm{mg} \mathrm{Cl} \cdot \mathrm{l}^{-1}$ (polished with paper of $80 \mu \mathrm{m}$ granulometry, passivated in air during $30 \mathrm{~min}$ ). 


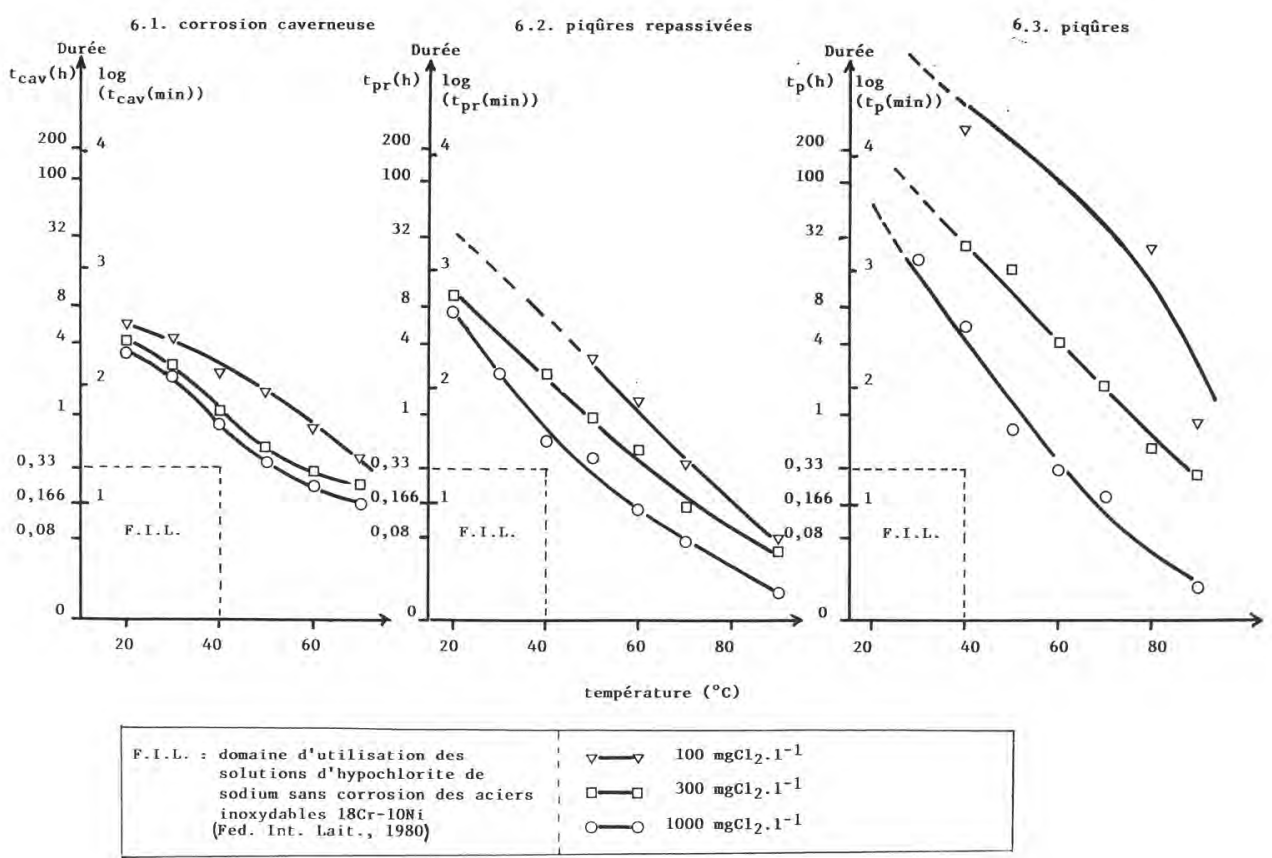

Fig. 6

Influence de la température des solutions agitées d'eau de javel sur la durée minimale avant corrosion de l'acier inoxydable $18 \mathrm{Cr} 10 \mathrm{Ni}$ (repère 15) (poli au papier de granulométrie $80 \mu \mathrm{m}$, passivé à l'air pendant $30 \mathrm{~min}$ ).

Influence of temperature of agitated sodium hypochlorite solutions on minimum time to corrosion of $304 \mathrm{~L}$ stainless steel (mark 15) (polished with paper of $80 \mu \mathrm{m}$ granulometry, passivated in air during $30 \mathrm{~min}$ ).

l'apparition de la corrosion. L'addition de dichromate de potassium dans la solution B ne modifie pas les durées avant corrosion (fig. 4) ni les potentiels.

La figure 5 regroupe les durées avant corrosion caverneuse et par piqûres, durées qui caractérisent la résistance des aciers inoxydables au contact d'une solution d'eau de javel de type A, à température élevée ( 70 ou $75^{\circ} \mathrm{C}$ suivant la forme de corrosion). Chaque acier ferritique (sans nickel) peut être comparé à un acier austénitique (contenant du nickel) homologue c'est-à-dire contenant le ou les mêmes éléments d'addition. On constate ainsi que pour les différents couples que l'on peut former ( 1 et $15-19 ; 6$ et $20 ; 5$ et 23 et enfin 7-8 et 28) la durée avant corrosion caverneuse est généralement plus courte pour les aciers ferritiques (fig. 5.1). Ce décalage n'existe plus pour les durées avant piqûres repassivées (fig 5.2) ni 
Fig. 7

Influence de l'agitation de la solution d'eau de javel (100 mg $\mathrm{Cl}_{2} \cdot 1^{-1}$ ) sur la durée minimale avant corrosion caverneuse de l'acier inoxydable $18 \mathrm{Cr} 10 \mathrm{Ni}$ (repère 15) (poli au papier de granulométrie $80 \mu \mathrm{m}$, passivé à l'air pendant $30 \mathrm{~min}$ ).

Influence of agitation of sodium hypochlorite solution (100 mg $\mathrm{Cl}_{2} . \mathrm{l}^{-1}$ ) on minimum time to crevice corrosion of 304 L stainless steel (mark 15) (polished with paper of $80 \mu \mathrm{m}$ granulometry, passivated in air during $30 \mathrm{~min}$ ).

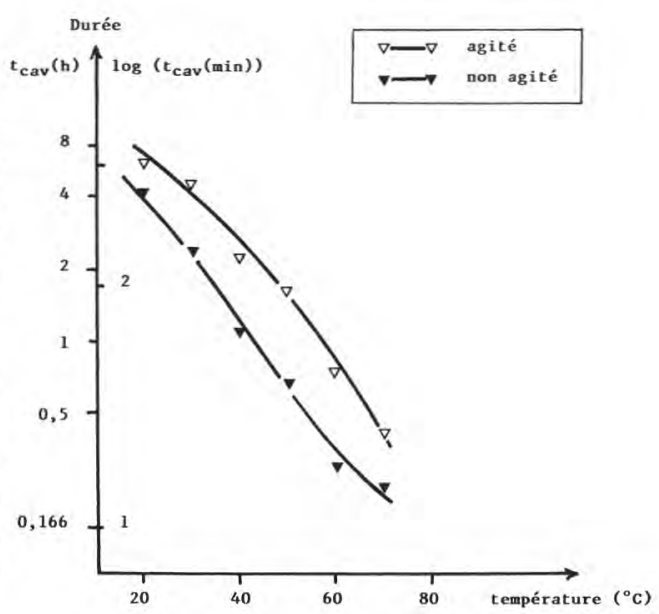

pour les durées avant piqûres que nous n'avons pas rapportées dans cet article. Enfin, dans les deux séries d'aciers, les durées avant corrosion dépendent de la nature et de la teneur en élément (s) d'addition : titane ou/et molybdène.

Pour l'acier $18 \mathrm{Cr} 10 \mathrm{Ni}$ (15), la durée minimale avant corrosion diminue très fortement lorsque la température s'élève (fig. 6). Il en est de même lorsque la concentration en hypochlorite augmente de 100 à $1000 \mathrm{mg} \mathrm{Cl} \mathrm{Cl}_{2} \mathrm{l}^{-1}$. Enfin, l'absence d'agitation réduit les durées avant corrosion. A titre d'exemple, les résultats concernant la corrosion caverneuse sont donnés sur la figure 7.

\section{DISCUSSION}

\section{Influence des ions présents dans la solution d'eau de javel}

Toutes les solutions corrosives (tab. 2) contenaient des ions chlorures $\left(\mathrm{Cl}^{-}\right)$, hydroxyle $\left(\mathrm{OH}^{-}\right)$et sodium $\left(\mathrm{Na}^{+}\right)$. Les solutions modèles D, E et F ne contenaient pas d'ions hypochlorite $\left(\mathrm{Cl0}^{-}\right)$; en outre, cette dernière ne renfermait pas d'ions chlorate $\left(\mathrm{ClO}_{3}\right)$ ou chlorite $\left(\mathrm{ClO}_{2}^{-}\right)$. Les ions $\mathrm{OH}^{-}$et $\mathrm{Na}^{+}$n'étant pas des agents de corrosion des aciers inoxydables, l'existence d'un potentiel critique (Ecav, Epr, Ep), c'est-à-dire d'un risque de corrosion, est entièrement imputable aux ions $\mathrm{Cl}^{-}$présents dans chacune de ces solutions. Les variations de concentration en ions $\mathrm{Cl}^{-}$ne modifient d'ailleurs pas la valeur du potentiel critique. Le rôle nocif de ces ions, reconnu pour tous les matériaux passivables comme les aciers inoxydables, explique les graves problèmes de corrosion rencontrés dans les 
milieux fortement chlorurés (eau de mer, " eaux » géothermales...) et la méfiance extrême manifestée à l'égard de tous les milieux contenant ces ions en concentration faible et apparemment anodine dans bien des secteurs d'activité (nucléaire, traitement de surface, automobile, bâtiment, pharmaceutique). Dans le secteur alimentaire lui-même, ces ions sont présents dans de nombreux produits et expliquent en particulier la corrosivité des lactosérum (Daufin et al., 1975) et même du lait ou des produits laitiers salés (Féd. Int. Lait., 1971) ou non (Trémoureux, 1978).

Le rôle joué par les ions $\mathrm{Cl0}^{-}$, présents en quantité appréciable uniquement dans la solution $A$, est celui d'un oxydant fort qui augmente très sensiblement le potentiel libre $E_{1}$ (fig. 3 ) jusqu'à des valeurs égales ou supérieures au potentiel critique Ecav ou Epr selon le cas entraînant ainsi la formation spontanée de la corrosion. La très faible concentration en ions $\mathrm{ClO}^{-}$de l'eau de javel vieillie (C) explique que cette solution ne soit pas plus agressive que la solution chlorurée de référence $(F)$. Le désaccord apparent de ce résultat avec celui de Botham (1949), concernant une solution d'hypochlorite plus corrosive après 6 mois de conservation, ne peut être expliqué faute d'éléments de comparaison identiques : évaluation de la corrosion par perte de masse; pas de données sur les concentratoins en ions présents.

Le dichromate, présent dans la solution B, est un oxydant fort mais sa concentration insuffisante $\left(0,73 \mathrm{mg} \cdot \mathrm{1}^{-1}\right)$ face à la teneur plus élevée en hypochlorite $\left(435,2 \mathrm{mg} \cdot \mathrm{1}^{-1}\right)$ n'entraîne aucune augmentation ni du potentiel libre ni des potentiels critiques (fig. 4) et ne modifie donc pas le pouvoir corrosif de la solution. La décision de ne plus ajouter ce colorant aux eaux de javel françaises, qui se justifie pleinement au plan de la toxicité, n'a donc aucune incidence au plan de la corrosion.

\section{Influence de la composition de l'acier inoxydable} (fig. 5)

Les résultats présentés dans ce travail illustrent bien la similitude de comportement de l'acier ferritique $17 \mathrm{Cr} \mathrm{Ti}$ (6) comparé aux 2 aciers austénitiques de référence $18 \mathrm{Cr} 10 \mathrm{Ni}$ (15 et 19). Des travaux antérieurs, consacrés à une plus vaste comparaison entre aciers austénitiques et ferritiques, ont montré que l'acier ferritique à $17 \% \mathrm{Cr}$ stabilisé au titane peut être envisagé pour la construction de matériel laitier soumis à des conditions de travail normales, peu sévères (Daufin et al., 1981). A cette fin, des opérations de prédéveloppement sont actuellement en cours et portent sur les conditions optimales de la soudabilité opératoire, la construction et la tenue en service de divers matériels pilotes.

En outre, dans les deux séries d'aciers, est mis en évidence le rôle favorable de l'addition de titane seul (6 et 20) plus marqué 
en piqûres qu'en caverne, de molybdène ( 5 et 23 ) plus fort en caverne qu'en piqûres et de ces deux éléments simultanément (7 et 28). Ceci justifie la reconnaissance, depuis quelques années, des aciers $17 \mathrm{Cr} 12 \mathrm{Ni} 2 \mathrm{MoTi}$ comme étant les aciers austénitiques les plus appropriés pour résoudre les cas de corrosion localisée les plus difficiles rencontrés dans le domaine alimentaire (traitement des lactosérum, cuves de salage en saumure...).

\section{Conditions limites d'utilisation des solutions d'eau de javel}

Afin de cerner la portée des résultats présentés mais aussi leur limite, il paraît essentiel de rappeler les conditions dans lesquelles nous avons décidé d'effectuer notre travail.

Ainsi, les essais de corrosion ont été réalisés avec l'acier $18 \mathrm{Cr}$ $10 \mathrm{Ni}$ (dans la nuance la plus couramment rencontrée pour le matériel alimentaire) ayant le repère 15 , c'est-à-dire celui dont la résistance à la corrosion localisée était la plus faible (en raison selon toute vraisemblance d'une teneur élevée, $0,010 \%$, en soufre) parmi une douzaine d'aciers de même nuance, issus de coulées différentes. En outre, pour accroître encore la marge de sécurité donnée à l'utilisation éventuelle des résultats présentés, seules sont données les valeurs minimales des durées avant corrosion. La prise en compte de ces éléments confère à nos essais une sévérité supérieure à celle que l'on peut attendre des conditions industrielles.

Cependant, la résistance à la corrosion dépend de trois autres facteurs importants, l'état de surface du matériau, la présence de zones soudées et le $\mathrm{pH}$ des solutions, dont l'influence n'a pas été explorée de façon systématique dans ce travail. Ainsi, divers états de surface (polis mécaniques plus ou moins fins ou grossiers, brut de laminage « glacé » ou « recuit brillant», passivation en solution nitrique plus ou moins concentrée, après décapage fluonitrique ou non,...) peuvent être rencontrés sur les matériels industriels dont la résistance à la corrosion peut être différente de celle de l'état de surface poli mécanique utilisé dans ce travail. En particulier, lorsque la rugosité créée par le polissage mécanique augmente, la résistance à la corrosion diminue (Daufin, 1974; Daufin et al., 1977 b). Les surfaces brutes de laminage "glacé » sont, quant à elles, plus ou moins résistantes que la surface polie de référence, selon la nature de l'acier et les conditions de température utilisées (Daufin et al. 1977 b; Lalande et al., 1981). Enfin, à notre connaissance, il n'existe pas de résultats concernant le comportement des surfaces brutes de laminage « recuit brillant». En conséquence, nous reprenons actuellement l'étude de ce problème dont les premiers résultats indiquent que l'acier $18 \mathrm{Cr} 10 \mathrm{Ni}$ (15) résiste mieux à la corrosion à l'état brut de laminage "glacé » qu'à l'état poli lorsque la température est inférieure à environ $50^{\circ} \mathrm{C}$. C'est l'inverse dans le domaine des températures élevées (Kerhervé et Daufin, 1983). 
Par ailleurs, nos résultats ne concernent que des surfaces ne présentant pas de soudure. Or, on sait que les risques de corrosion sont beaucoup plus importants sur ou au voisinage de soudures qui ne sont pas réalisées dans les règles de l'art et qui ne sont pas soigneusement usinées, polies et éventuellement décontaminées (Fed. Int. Lait., 1981).

Enfin, le $\mathrm{pH}$ des solutions était celui obtenu par dilution de l'extrait de javel dans l'eau : à $25^{\circ} \mathrm{C}, \mathrm{pH}=9,3 ; 9,7$ et 10,6 à respectivement 100,300 et $1000 \mathrm{mg} \mathrm{Cl}_{2} \cdot \mathrm{l}^{-1}$. Or, la modification du $\mathrm{pH}$ consécutive, par exemple, à un mauvais rinçage ou à une addition malencontreuse ou volontaire d'une base ou d'un acide, modifie le pouvoir corrosif de la solution désinfectante. En milieu acide, en particulier, les risques de corrosion sont beaucoup plus grands sur les surfaces émergées (Kerhervé et Daufin, 1983).

Les résultats exposés sur les figures 6.1 (corrosion caverneuse), 6.2 (piqûres repassivées) et 6.3 (piqûres) montrent que pour les trois concentrations étudiées : 100,300 et $1000 \mathrm{mg} \mathrm{Cl}_{2} \cdot 1^{-1}$, il existe un large domaine de couples température-durée de contact n'entrâ̂nant l'apparition d'aucune corrosion.

Le domaine relatif à l'absence de corrosion caverneuse est plus restreint que celui relatif à la corrosion par piqûres, élément qui doit inciter les constructeurs à dessiner et concevoir leurs installations sans interstices susceptibles d'être des sites privilégiés de corrosion caverneuse.

Pour une forme de corrosion donnée, l'étendue du domaine sans corrosion diminue lorsque la concentration en eau de javel augmente, conséquence logique de l'augmentation du pouvoir oxydant du milieu (il y a plus d'ions $\mathrm{Cl0}^{-}$) et à un degré moindre de la teneur en ions agressifs $\mathrm{Cl}^{-}$. L'absence d'agitation a un effet similaire (fig. 7) d'autant plus fort que la concentration en eau de javel est plus faible. Ainsi, les durées avant corrosion sont divisées par environ 2 ; 1,$5 ; 1,25$ respectivement aux concentrations de 100,300 et $1000 \mathrm{mg}$ $\mathrm{Cl}_{2} \cdot \mathrm{1}^{-1}$. Le rôle très complexe de l'agitation s'explique, en partie, par son effet sur les couches limites et donc sur les phénomènes de diffusion dont la vitesse contrôle le transfert des espèces depuis et vers les sites de corrosion.

Enfin, les durées avant corrosion diminuent très fortement lorsque la température croît de 20 à $90^{\circ} \mathrm{C}$. Cette tendance confirme les résultats de Botham et Dummet (1949), Rossman (1964), Cowan (1971) et Daufin (1977) et est en accord avec toutes les données connues concernant l'influence de la température sur l'apparition de la corrosion localisée.

Sur un plan pratique, on constate que notre travail définit des domaines d'utilisation des solutions d'eau de javel sans corrosion beaucoup plus larges que le domaine défini dans le document $n^{\circ} 127$ de la Fédération Internationale de Laiterie (1980) et reporté sur la figure 6. Les domaines ainsi définis doivent permettre à l'industriel 
alimentaire d'éviter la corrosion de ses installations même dans des cas spécifiques où il est contraint d'appliquer un traitement à température élevée et/ou à forte concentration et/ou pendant une durée longue. En particulier, dans la lutte contre les bactériophages, l'utilisation de concentrations élevées (1000 $\mathrm{mg} \mathrm{Cl}_{2} \cdot 1^{-1}$ ) pendant quelques dizaines de minutes, indispensable pour assurer un effet destructeur vis-à-vis de ces virus, nécessite d'opérer à basse température. Indiquons en outre qu'une désinfection classique satisfaisante est obtenue en une durée maximale de 15 à $20 \mathrm{~min}$ et que les solutions d'eau de javel sont plus stables et donc plus efficaces à 20 qu'à 40 ou $60^{\circ} \mathrm{C}$ (Sainclivier, 1964 ; Kerhervé et Lostanlen, 1969).

En conséquence, si dans ces conditions optimales de désinfection, la solution ne présente pas l'effet bactéricide attendu, c'est que l'eau de javel ne peut pas détruire les micro-organismes concernés (hors du spectre bactéricide, hors d'atteinte du produit : recoins, dépôts, interstices...) et ne pourra pas y parvenir même si on change les conditions de travail. Ce changement aura pour seul effet d'aggraver les risques de corrosion.

\section{CONCLUSION}

La présence conjointe d'ions agressifs (chlorure : $\mathrm{Cl}^{-}$) et d'ions fortement oxydants (hypochlorite : $\mathrm{Cl}^{-}$) dans les solutions d'eau de javel est susceptible de provoquer l'apparition et le développement de corrosion caverneuse et par piqûres sur les aciers inoxydables. La suppression de l'addition de dichromate de potassium à partir de la fin de l'année 1976 n'a pas modifié le pouvoir corrosif de ce milieu désinfectant.

Les nuances ferritiques et austénitiques les plus chargées en éléments d'addition, molybdène, titane et surtout ces deux éléments simultanément, résistent mieux à la corrosion que la nuance classique $18 \mathrm{Cr} 10 \mathrm{Ni}$ et la nuance ferritique $17 \mathrm{Cr} \mathrm{Ti}$ qui lui est sensiblement équivalente.

Pour l'acier austénitique de référence $18 \mathrm{Cr} 10 \mathrm{Ni}$ (15) nous avons défini de larges domaines de conditions température-duréeconcentration sans danger de corrosion. L'augmentation de la valeur de l'un de ces facteurs réduit l'étendue de ces domaines. Pratiquement, en considérant simultanément l'efficacité bactéricide et les risques de corrosion, on aboutit aux préconisations générales d'emploi suivantes, valables pour des concentrations jusqu'à $1000 \mathrm{mg}$ $\mathrm{Cl}_{2} \cdot 1^{-1}$ :

- durée d'action inférieure à $30 \mathrm{~min}$;

- température inférieure à $30^{\circ} \mathrm{C}$.

Le respect de cette température critique concerne la solution désinfectante d'eau de javel non seulement lors de sa préparation mais aussi et surtout au niveau de la paroi du matériel à désinfecter, 
en particulier des échangeurs de chaleur (qui doivent être refroidis avant la désinfection).

\section{Bibliographie}

Botham (G. H.), Dummet (G. A.) (1979). - Corrosion by commercial sodium hypochlorites and its inhibition. J. Dairy Res., 16, 23-38.

Вотнам (G. H.) (1962). - The corrosion resistance of stainless steels to the new organic sterilizers containing chlorine. XVI Cong. Int. Lait., C, 167-175.

Castang (Mme), Cauchois (Mlle), Soulès (Mme), M. M. Ansaloni, Domange, FloRentin, LipPs, Ney, Nortz, Prost, Saulnier et Sultan (1965). - Rétrogradation des eaux et extraits de javel. Ann. Fals. Exp. Chim., 665, 49-57.

CoWAN (C. T.) (1971). - Corrosion and its prevention in dairy process plant. J. Soc. Dairy Technol., 24 (2-3), 90-95.

Daufin (G.) (1974). - Etude de quelques cas de corrosion d'alliages utilisés dans l'industrie laitière et de l'influence de l'état de surface sur leur propreté bactériologique. Thèse de doctorat d'Etat. Paris.

Daufin (G.), Kerhervé (L.), Michel (Françoise) (1975). - Corrosion de diverses nuances d'aciers inoxydables par le lactosérum. Ind. Alim. Agric., 5, 495-604.

DaufiN (G) (1977). - Etude de la corrosion par piqûres d'un acier inoxydable du type $18 \% \mathrm{Cr}-10 \% \mathrm{Ni}$ par des solutions de désinfection à base d'hypochlorite de sodium. Ind. Alim. Agric., 6, 577-578.

Daufin (G.), Desestret (A.), Kerhervé (L.), Michel (Françoise), Trémoureux (Y.), VAllier (G.) (1977a). - Etude de la corrosion d'aciers inoxydables ferritiques et austéniques et d'alliages à hautes caractéristiques mécaniques dans les conditions de l'industrie laitière. C.R. fin d'étude DGRST, 75.7.1139 et 1140 .

Daufin (G.), Kerhervé (L.), Richard (J.) (1977b). - Influence de l'état de surface de l'acier inoxydable sur son aptitude au nettoyage et sur sa sensibilité à la corrosion par piqûres. Ind. Alim. Agric., 1, 43-51.

Daufin (G.), Desestret (A.), Kerherivé (L.), Vallier (G.) (1979). - Etude de la corrosion d'aciers inoxydables ferritiques et austénitiques et d'alliages à hautes caractéristiques mécaniques dans les conditions de l'industrie laitière. C.R. fin d'études D.G.R.S.T., 77.7.0701 et 0702 .

Daufin (G), Desestret (A.), Kerhervé (L.), Michel (Françoise), Vallier (G.) (1981). - Utilisation des aciers ferritiques à $17 \% \mathrm{Cr}$ dans l'industrie laitière. Mém. Sci. Rev. Métall., 78 (12), 647-662.

Daufin (G.), Kerhervé (L.), Michel (Françoise), Desestret (A.), Vallier (G.) (1982). - Les critères de résistance à la corrosion et la sélection des aciers inoxydables dans les industries alimentaires. Métaux Corros. Ind., 57 (682), 213-225.

Defranoux (J.M.) (1968). - Sur le comportement des aciers inoxydables en présence d'eau de mer froide et chaude. Corros. Sci., 8, 245-258.

Féd. INT. LaIt. (1971). - Corrosion métallique en laiterie. Bull. ann., partie 3.

FÉD. InT. LAIT. (1980). - Corrosion en industrie laitière. Doc. 127.

Féd. Int. LaIt. (1981). - Corrosion dans l'industrie laitière. Doc. 139 (brochure no 3).

Journal Officiel (1968). - Méthodes officielles d'analyse des eaux et extraits de javel, 9491-9492, 8 octobre.

KeRHervé (L.), Lostanlen (D.) (1969), - Essais de détergents-désinfectants de laiterie. Rev. Lait. Franç., 263, 165-175.

Kerhervé (L.), Daufin (G.) (1983). - Résultats non publiés. 
Lalande (M.), Corrieu (G.), Daufin (G.), Gallot-Lavallée (T.) (1981). - Etude des cinétiques d'encrassement et de nettoyage d'un pasteurisateur de lait à plaques. C.R. fin d'études D.G.R.S.T., 79.7.0375.

Rossman (C. H. R.) (1964). - Beständigkeit von austenitischem chrom nickel-stahl gegen Lochfrass bei natriumhypochlorithaltigen Reinigungs-unds desinfektionsmitteln. Nickel Berichte, 22, 28-32.

Sainclivier (M.) (1964). Evaluation de la capacité désinfectante de quelques produits utilisés en laiterie. Ind. Lait., 206, 2-6, 207, 51-66.

SHÈre (L.), ChandLer (R. E.), WALters (A. M.) (1965). - Laboratory evaluation of bacterial efficiency and corrosion activity of hypochlorites on stainless steels. Int. Cong. Food Sci. Microbiol., 11, 499-505.

SPEIGHT (G. E.) (1957). - Dairy Technol., 1, 17.

Trémoureux (Y.) (1978). - Etude de la résistance à la corrosion d'alliages à hautes caractéristiques mécaniques dans quelques milieux de l'industrie laitière. Thèse de docteur ingénieur, Paris 6. 